\title{
Conceptualizing Institutions And Organizations: A Critical Approach
}

Natalia Boliari, Manhattan College

Kudret Topyan, (E-mail: kudret_topyan@yahoo.com), Manhattan College

\begin{abstract}
This paper reviews the concept of institutions and its relationship to capitalist development as used in the field of NIE and compares it to the use of the concept by Hall and Soskice. By specifying exactly what institutions are, how and why they should be differentiated from organizations, and how they influence transaction and production costs, Douglass North and the NIE articulate the role of institutions in understanding economic development. Hall and Soskice however, reveals a broad and varied use of the concept which ignores one of the key elements of institutions - enforcement and also fails to provide a clear differentiation between institutions and organizations which leads to confusions in understanding their specific roles in the process of development of the two types of economies that the authors define. A varied, imprecise and inconsistent use of the concept may lead to an overestimation of the role of institutions and conversely underestimation of the role of organizations in capitalist development.
\end{abstract}

\section{INTRODUCTION}

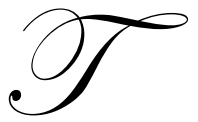

he terms 'capitalism' and "capitalist development” have been with us for many years. For more than a century, scholars from different disciplines have provided different analytical frameworks, theories, and models of capitalist development that are products of decades of deep reflection and research. These theories and models have sought to explain and predict why and how different capitalist economies develop or not develop over time; "why capitalism triumphs in the West and fails everywhere else" and related to this, what are the critical factors, endowments, policies or arrangements that have led to its success in the West. Since the concepts represent an ever evolving multifaceted and complex process, each theory has only been able to provide insights into a few dimensions and it is not surprising that there is no universally agreed theory of capitalist development.

The institutional approach is considered as one of the most plausible of the post-Second World War theories of development (Preston, 1996). What makes it different from other approaches is that, it examines problem-specific formulations using analytical tools - the materials of sociology, politics, anthropology, history, and others. The importance of the New Institutional Economics (NIE) has been confirmed with the acclaimed and awarded works of Ronald Coase and Douglass North and consequently the role of institutions for development has received considerable attention from economists, development researchers, policy makers, and many others (Harriss et al., 1995). The contextual framework developed by the NIE has inspired the analysis and works of many researchers form different disciplines. However, in the social sciences the concept of institutions has been used broadly and in a variety of ways and often the literature has failed to provide a clear context of the concept, leading to controversies and confusion for the reader.

This paper aims to review the concept of institutions and its relationship to capitalist development as used in the field of the NIE on one hand, and as used by Peter Hall and David Soskice in their Varieties of Capitalism, on the other. Accordingly, the paper is organized in four sections: the first provides a brief overview of the NIE - the multidisciplinary field that questions and examines the role of institutions in development; the second defines and analyzes the concept of institutions as used within the framework of the NIE, focusing on the works of North; the third examines the relationship of institutions to economic development; and the fourth analyzes the use of the concept by Hall and Soskice. The paper argues that drawing on concepts developed by the NIE and on the definition of 
institutions provided by North, Hall and Soskice construct a broad and loose conception of institutions which makes it difficult for the student to differentiate between the impacts of institutions and organizations and exactly understand the role of institutions in their analysis of comparative capitalism.

\section{An Overview of the New Institutional Economics (NIE)}

The NIE has been broadly perceived as an alternative to mainstream neoclassical economic theory. Contrary to this belief, North (in Harriss et al., 1995) states that "the NIE builds on, modifies, and extends neoclassical theory to permit it to come to grips and deal with an entire range of issues heretofore beyond its ken". In other words, the NIE can be described as an analytical system born within the frame of neoclassical economics, but offering answers to many of its "puzzles" by modifying and extending it. Harriss et al. (1995) emphasize the significance of the NIE by pointing out the importance of three of its characteristics: (1) it is a major development within the dominant paradigm of modern economics since it provided an answer to the puzzle of why the firm exists as an administrative and financial organization and clarified the role of transaction costs in neoclassical economic theory; (2) it has questioned and challenged the dominant role that the neoclassical orthodoxy has assigned to the market; and (3) it provides a theory of development in terms of appropriate reorganization and change of institutions with the aim of promoting and fostering economic growth.

There are five branches existing within the NIE: transaction cost economics, the economics of imperfect information, the economics of property rights, collective action theory, and the evolution of rules and norms (Clague et al., 1997). The NIE aims to explain the determinants of institutions and their evolution over time as well as to evaluate their impact on economic performance, efficiency and distribution (Nabli and Nugent, 1989). To achieve this, the NIE incorporates within its framework some aspects of the social sciences - history, anthropology, sociology, law, political science, and business organization and thus can be described as a multi- and interdisciplinary approach to development.

According to the NIE, institutions are the way in which economies cope with "market failure"; the Paretoefficient outcome of neoclassical economics is only one of many possible outcomes; and an economy will only achieve the efficient outcome under certain institutional arrangements (Yeager, 1998, pp. xiv). While keeping the assumption of the neoclassical orthodoxy that self-seeking individuals attempt to maximize objectives under scarcity and constraints, the NIE "relaxes" the assumptions of full rationality, zero transaction costs, and perfect information. With this new direction, the costs of transacting which are determined by institutions and institutional arrangements are considered as the key to economic performance. Therefore, an argument is made that the institutions of a country such as its legal, political, and social systems are first order determinants of its economic performance, and this is what makes the new institutional economics important for researchers from different disciplines.

\section{What Are Institutions?}

The most commonly agreed upon definition of institutions is provided by North (1990, pp. 3): "Institutions are the rules of the game of a society, or, more formally, are the humanly devised constraints that structure human interaction. In consequence they structure incentives in human exchange, whether political, social, or economic.” The key word in the definition of North is constraints - institutions include any form of constraint that human beings devise to shape human interaction. According to the author, institutions are composed of formal rules created by human beings such as statute law, common law, and regulations; informal constraints such as conventions, norms of behaviour and self imposed codes of conduct; and the enforcement characteristics of both. The definition suggests three fundamental elements of institutions: The first one is the formal or written rules - political systems, laws governing contracts, crime, product information, the imposition of taxes, tariffs, regulation of banks, universities, etc. As such, they can be created by governments as well as within firms and other organizations. The second one is the informal or unwritten rules - culture, norms of behaviour, customs, values, religions, etc. They are generated from socially transmitted information and imposed by people upon themselves in order to structure their relationships with each other. The last one is the enforcement mechanisms - institutions are ineffective when they are not enforced. Enforcement mechanisms are an integral part of the institutional framework of a society and can function fully, 
marginally, or not function at all. According to Yeager (1998, pp. 10) they can be "the single most important element in explaining differences in economic performance."

North emphasizes that the major role of institutions is "to reduce uncertainty by establishing a stable (but not necessarily efficient) structure to human interaction" and points out that both formal and informal institutions are evolving and changing, thereby continually altering the choices available to us (1990, pp. 6). The change in institutions occurs incrementally since it is a consequence of the imbeddedness of informal constraints in societies. While the change in formal rules (as a result of political or judicial decisions) may occur as fast as overnight; "informal constraints embodied in customs, traditions, and codes of conduct are much more impervious to deliberate policies" (1990, pp. 6). These cultural constraints represent the link between the past and the future and provide "the key to explaining the path of historical change.” Therefore, in here, one will see a representation of the complex interaction between the State as a designer of formal rules and the society as being bounded by its informal constraints.

North draws special attention to the definition of organisations: They are the actors or players -groups of individuals bound by a common purpose to achieve objectives (1990, pp. 5) while institutions are the rules of the game of a society. These players can be "political bodies (political parties, the Senate, a city council, a regulatory agency); economic bodies (firms, trade unions, family farms, cooperatives); social bodies (churches, clubs, athletic associations); and educational bodies (schools, universities, vocational training centres)." Accordingly, modeling organizations requires analyzing governance structures, skills, and the way learning by doing will determine the organization's success over time. Therefore, the way organizations come into existence and the way they evolve are fundamentally influenced by the institutional framework of a society.

North, by emphasizing the interaction between institutions and organizations, further draws attention to the role of institutions as the underlying rules of the game on one hand and the role of organizations (and their entrepreneurs) as agents of institutional change on the other. Moreover, North advocates "separating the analysis of the underlying rules from the strategy of the players" as an absolute prerequisite to building a theory of institutions (1990, pp. 5). Just like institutions, organizations provide a structure to human interaction. However, the author emphasizes that, "Conceptually, what must be clearly differentiated are the rules from the players. The purpose of the rules is to define the way the game is played. But the objective of the team within that set of rules is to win the game by a combination of skills, strategy and coordination; by fair means and sometimes by foul means" (1990, pp. 4-5). Yeager (1998, pp. 10-11), furthermore, notes that the distinction between institutions and organizations is subtle but crucial and it has to be clear for a proper understanding of the role of institutions. Organizations themselves are not institutions; they operate under the institutional framework of a society but they also create their own formal and informal institutions and the enforcement mechanisms of both.

\section{Institutions and Their Relation to Economic Growth and Development}

North argues that "institutions affect the performance of the economy by their effect on the costs of exchange and production. Together with the technology employed, they determine transaction and transformation (production) costs that make up total costs...The costliness of information is the key to the costs of transacting, which consists of the costs of measuring the valuable attributes of what is being exchanged and the costs of protecting rights and policing and enforcing agreements. These measurement and enforcement costs are the sources of social, political, and economic institutions" (1990, pp.5-6; pp. 27). Limited information and limited computational ability lead to the creation of informal constraints, which in turn leads to reduction in the costs of human interaction (1990, pp. 36). On the other hand, the effectiveness of informal constraints is enforced by formal rules which in turn, would lower information, monitoring, and enforcement costs (1990, pp. 46). The problem with the concept of enforcement is that it is not given and it is neither perfect, nor constantly imperfect as it is generally assumed in the literature. According to North, the structure of enforcement mechanisms and the frequency and severity of imperfection greatly influence the costs of transacting and the forms that contracts take (1990, pp. 54).

Theoretical, historical, and cross-country evidence suggests that informal institutions affect performance substantially and thus "they should facilitate economic exchange both by supporting self-enforcing rules of the game 
and by fostering trust in third-party enforcement through the state (Raiser, 2001). Raiser schematically represents the interrelationships between formal and informal institutions where he demonstrates that the presence of a specific type of moral norms leads to the emergence of a civil society the existence of which in turn reinforces the moral predispositions of individuals and creates 'trust' among economic actors. This consequently leads to the "facilitation of economic exchange under imperfect information, and that trust and cooperative behaviour grow out of an articulated civil society and depend positively on the existence of a universal morality at the nationwide level".

Drawing on North's theory, Yeager (1998, pp. 35-54) illustrates how institutions affect economic performance using two models: the 'static' case where the level of technology is held constant and the 'dynamic' case where technology advances and leads to an increase in economic wealth.

The Static Case performance:

By working backwards, from right to left, Yeager demonstrates the impact of institutions on economic

\section{Institutions $\rightarrow$ Transaction Costs $\rightarrow$ Creation of Markets $\rightarrow$ Specialization and Division of Labour $\rightarrow$ Productivity $\rightarrow$ Economic Performance}

He indicates that for markets to exist and function well, there must be at least six criteria in place: consumer demand; suppliers; good information for consumers regarding prices, quality, and attributes of the products; stable monetary system recognized and trusted by both the buyers and suppliers; legitimate and secure seller's ownership of the property rights to the good or service and authority to transfer these rights to the buyer; and access for both buyers and sellers to an impartial legal system "in case one party cheats on the other". North and Yeager identify three types of markets:

1. Personal exchange with no third party enforcement - exchange is conducted between relatives and community members which rely and trust on each other

2. Impersonal exchange with no third party enforcement - described as having very high transaction costs since no explicit bonds exist between the parties to the exchange

3. Impersonal exchange with third party enforcement

The authors argue that it is the third type of market structure that is absolutely essential for the solid performance of modern capitalist economies. Only in this type of structure will individuals accept taking the risks inherent in exchange with people they do not know very well. Accordingly, North and Yeager indicate that wellfunctioning markets require low transaction costs and these costs can be determined by a nation's institutional framework. Based on the static model, Yeager emphasizes that formal rules must clearly define the property rights to the goods or services to be exchanged; the informal rules must promote a sense of trust and respect for the rule of law, and finally enforcement must be carried out as objectively and fairly as possible. It is suggested that the institutional framework of a nation determines the level of transaction costs, which in turn determines how well markets function, which in turn determines economic performance.

\section{The Dynamic Case}

With the 'dynamic' model where technology is advancing, Yeager demonstrates how institutions affect economic performance through time.

\section{Institutions $\rightarrow$ Behaviour of Organizations $\rightarrow$ Process of Creative Destruction $\rightarrow$ Technological Progress $\rightarrow$ Economic Wealth}

The author addresses three crucial elements of the extremely complex process of technological development: (1) promoting the growth of human creativity which requires openness to change and willingness to deal with the disruption of the status quo brought by new technology (here Yeager relates openness to change to the informal 
constraints of society); (2) the presence of a well-functioning capital markets which require low transaction costs; and (3) a competitive environment forcing firms to continually improve their products (pp. 47-49). He notes that "the process of creative destruction does not occur in every economy, but only in one where the proper institutional framework is present” (pp. 50). The key role assigned here to institutions is to constrain firm's activities enough so that "the main way for them to make profits is by increasing technology". The author also emphasizes that institutions must constantly evolve to respond to changing circumstances (e.g. by protecting intellectual property rights), foster creativity, lower transaction costs, and encourage the process of creative destruction.

Accordingly, it is argued that low economic performance and consequently the persistence of poverty is linked to three primary reasons: (1) the "path dependency" characteristic of economies - once an economy is on a certain path, it is very difficult to change it. Individuals and organizations adapt to existing institutional structures and resist changes; creators of formal rules benefit from the status quo; (2) the gap between the world's complexity and people's ability to understand it - uncertainty and lack of perfect information. Scholars still do not clearly understand the complex interactions between institutions and economic performance; thus it is not exactly known what advice is to be given to countries with problems; and (3) the slow evolution of informal institutions - as the cultural traits are passed from generation to generation, economic performance tends to repeat itself. It may take decades and even centuries for informal institutions to evolve into traditions promoting economic growth (Yeager, 1998, pp. 53).

\section{Varieties of Capitalism}

In Varieties of Capitalism, Hall and Soskice (2001) compare a number of developed economies by developing a framework for understanding their institutional similarities and differences. Their approach is based on three previously developed perspectives on institutional variation used in the study of comparative capitalism: the modernization, neo-corporatism, and social systems of production approaches. The novelty brought by Hall and Soskice is that their analysis goes beyond these approaches by placing the firm in the very centre of their analysis and by focusing on the organization of the private sector and variations among national political economies. The authors note that the fundamental difference of their analysis is in their "conception of how behaviour is affected by the institutions of the political economy" (2001, pp. 4). According to this conception, the behaviour of economic actors and political and economic outcomes are determined by strategic interactions conditioned by certain institutions which distinguish one political economy from another (2001, pp. 5).

Hall and Soskice distinguish between two types of developed political economies: liberal market economies (LMEs) and coordinated market economies (CMEs). In LMEs (e.g. USA) firm's activities are coordinated "via hierarchies and competitive market arrangements", that is, demand and supply conditions determine the equilibrium outcomes of firms' behaviour. On the other hand, firms in CMEs (e.g. Germany) coordinate their activities based on non-market relationships and therefore their coordination equilibrium is an outcome of their strategic interaction with other actors (2001, pp.8).

Hall and Soskice develop their approach centering on the coordination problems of firms whose "capabilities are ultimately relational" and whose success, according to the authors, "depends substantially on [their] ability to coordinate effectively with a wide range of actors" (2001, pp. 6). Accordingly, for each type of economy the authors explore five spheres which require the development of relationships necessary for the resolution of coordination problems: industrial relations, vocational training and education, corporate governance, inter-firm relations, and relationships with employees (2001, pp. 6-7). Hall and Soskice advance a thesis according to which the similarity between these two economies is in their institutional coherence which leads to the generation of good macroeconomic outcomes regardless of the difference in their institutions; whereas the fundamental difference is in the characteristics of the institutional complementarities present in these economies.

Institutional complementarity is defined as the situation where "the presence (or efficiency) of one [institution] increases the returns from (or efficiency of) the other" (pp. 17). An example of outcome of institutional complementarity between institutions of different spheres would be the feasibility of long-term employment resulting from a financial system which provides capital on terms insensitive to current profitability. In the reverse case "fluid 
labour markets may be more effective at sustaining employment in the presence of financial markets that transfer resources readily among endeavours thereby maintaining a demand for labour” (2001, pp. 18).

Institutional complementarities are found to be relevant to the study of the varieties of capitalism since their existence "suggests that nations with a particular type of coordination in one sphere of the economy should tend to develop complementary practices in other spheres as well.” The hypothesis is supported to some extent by the findings of institutional complementarities in the most highly developed OECD countries; six of them are classified as LMEs, ten as CMEs, and the remaining six "are in a more ambiguous position" (2001, pp. 19-21). Consequently, the authors argue that institutions and their complementarities have different effects on the two types of political economies such as generating different capacities for innovation, different distributions of income and employment and therefore, different distributions of well-being.

Accordingly, Hall and Soskice compare the institutional complementarities, institutional coherence, and coordination mechanisms present in the five spheres of different developed economies. They find that LMEs and CMEs differ in terms of "the extent to which firms rely on market mechanisms to coordinate their endeavours as opposed to forms of strategic interaction supported by non-market institutions.” They also point out that there are differences in terms of types of coordination among the LMEs and the CMEs and that each economy has specific capacities for coordination which determine the actions of both firms and governments (2001, pp. 34-35). According to the authors, coordination is present in both types of economies and differences in coordination result from differences in the institutions of each economy.

Furthermore, Hall and Soskice argue that the varieties of capitalism approach can be applied in the process of understanding comparative economic advantage and introduce the concept of comparative institutional advantage (CIA). According to the authors, each institutional structure provides different advantages for engaging in different activities. The more efficient production of some goods compared to the production of others is a result of the specific institutional support firms receive for performing the activities necessary for the production of these goods. The argument of the authors is that the institutions supporting these activities "are not distributed evenly across countries" (2001, pp. 37). Furthermore, the authors focus on the impact of CIA on innovation and argue that it determines the type of innovation in the two types of economies. Accordingly, they argue that institutional features of LMEs' support and provide better capacities for radical innovation and limit incremental innovation; whereas those of CMEs support and provide better capacity for incremental innovation and limit radical innovation (2001, pp. 36-41).

The varieties of capitalism approach taken by Hall and Soskice argues that successful macroeconomic performance depends on institutional coherence, that is, there is a functional relationship between the two. This follows from the argument that "...the economy is an arena in which multiple actors develop competencies by devising better ways of coordinating their endeavours with one another. When firms coordinate more effectively, their performance will be better, and the result will be better overall economic performance" (2001, pp. 45). Therefore, the authors suggest that national economic performance can be improved with policies promoting better forms of coordination among private-sector actors.

It can be argued that using the analytical lens of the concept of institutions, Hall and Soskice provide an impressive and sophisticated framework which sheds light into the discussion of comparative capitalism, the varieties of institutions and institutional structures capitalism takes across developed political economies, and the impact of institutions on these economies. However, the concept of institutions in Hall and Soskice's Varieties of Capitalism has a varied and somewhat imprecise content which can be interpreted in a variety of ways thereby leading to confusions and lack of clarity as to how exactly institutions affect the development of capitalism. The authors define the concept in a very broad and almost all-inclusive manner embracing almost everything that is important in the environment and for the proper functioning of their central object - the firm. Such a use of the concept can easily lead to the conclusion that indeed, institutions do matter but without being clear on exactly why and how they matter.

Given the broad use of the concept of institutions in the social sciences, one of the first things that the reader expects is to see a clear definition which would give her an idea of how the concept is going to be applied in the analysis of question. Following a description of the two types of political economies, Hall and Soskice define 
institutions as follows: "Institutions, organizations, and culture enter this analysis because of the support they provide for the relationships firms develop to resolve coordination problems. Following North (1990:3), we define institutions as a set of rules, formal or informal, that actors generally follow, whether for normative, cognitive, or material reasons, and organizations as durable entities with formally recognized members, whose rules also contribute to the institutions of the political economy. From this perspective, markets are institutions that support relationships of particular types, marked by arm's-length relations and high levels of competitions” (pp. 9).

While referring to North, the authors actually deviate from his definition. For North, institutions structure and shape human behaviour, interaction, and relationships by constraining them and consequently structuring incentives in human exchange; whereas for Hall and Soskice, institutions are there to support specific relationships between actors along with organizations and culture. Hall and Soskice define firms as actors and their definition can be interpreted as if actors were there before institutions and created the institutions for a specific purpose - to support relationships resolving coordination problems. However, North emphasizes that the way organizations (firms are organizations) come into existence and the way they evolve are fundamentally influenced by the institutional framework of a society.

At first glance, North's definition sounds like a broader one since it starts with the individual and encompasses the society as a whole. However, by clarifying the meaning of the three important components of institutions, North actually gradually elucidates the role of institutions in the complex formation and evolution of the relationships between the state, society, and the economy as a whole. The definition of Hall and Soskice, on the other hand, sounds at first like a narrower one since they focus on the impact of institutions on the activities of the firm. The problem arises later in the analysis, when the authors use the concepts of institutions, organizations, and even strategic actions interchangeably.

Secondly, in their definition and throughout the analysis, Hall and Soskice never mention the third important component of institutions and its role in the development of the varieties of capitalism - their enforcement mechanisms. Therefore, it can be argued that, just as in the general literature, the authors assume that enforcement is implicitly there by definition and necessarily takes place just because of the presence of institutions. Their definition also leads to the interpretation that actors somewhat voluntarily follow the formal and informal rules for "normative, cognitive, or material reasons". This is a fundamental divergence from the concept of the NIE. As noted by Yeager, enforcement and its mechanisms are considered as a key element in explaining differences in economic performance since they greatly influence the costs of transacting and the forms that contracts take. Therefore, ignoring the structure of enforcement mechanisms, the frequency and severity of their perfection or imperfection is an important drawback in the analysis of Hall and Soskice. Moreover, measurement and enforcement costs are themselves sources of social, political, and economic institutions; the lack of an enforcement analysis ignores the role of enforcement costs in shaping and structuring the institutions of LMEs and CMEs.

Thirdly, as discussed and emphasized in Section 2 of this paper, North and the NIE make a clear and well justified argument for the need to distinguish between institutions and organizations highlighting that this distinction is crucial in the construction and understanding of the theory of institutions and its application. Hall and Soskice indicate that they follow North's definitions for institutions and organizations; but throughout the analysis they ignore the distinction between institutions and organizations and often use the terms interchangeably. As can be understood from footnote 9, Hall and Soskice do this intentionally but the reason for this is not justified and leads to a contradiction between the definition and the footnote and later to confusions in the approach. ${ }^{1}$ Throughout their analysis, the authors often refer to institutions as associations, unions, networks, organizations, firms, etc. (e.g. pp.10 first paragraph). Such use of the concept of institutions leads to a confusion in terms of differentiating and understanding the exact role and impact of both institutions and organizations in the development of capitalism as well as the types of relationships that exist between them. Here we see the varied, broad, and all-inclusive meaning that is assigned to institutions which makes it difficult to understand the existing causal relationships and no wonder in such a case ‘institutions' can be deemed more important than they actually are.

\footnotetext{
${ }^{1}$ Footnote 9 on pp. 9 states: “Note that, from time to time, we refer loosely to the 'institutions' or 'organization' of the political economy to refer to both the organizations and institutions found within it."
} 
Fourthly, in economics and in the framework of the NIE markets represent a system of various commercial activities which require certain type of supporting institutions (legal framework) to facilitate their proper functioning. Although influencing each other, here activities are distinguished from institutions. For Hall and Soskice, markets themselves are institutions that support particular relationships and are accompanied by a legal system to support formal contracting. This description is again problematic since it is not clear why markets are institutions and if so, exactly what kind of institutions (rules) they are.

Finally, the NIE defines culture as a type of informal institution and as such it is a constraint that shapes human interaction and behaviour. However, culture enters the analysis of Hall and Soskice as a separate entity, "a set of shared understandings or available 'strategies for action' developed from experience of operating in a particular environment...” (pp. 13). Defining culture as a set of 'strategies for action' can be considered as implausible since strategy requires the presence of an objective and a plan for achieving that objective. However, usually culture is taken as a given by the members of a society, it does not change easily and it actually directs the actions of actors by constraining these actions not necessarily with their own will. In other words, strategy implies a free choice, whereas culture restricts the choices of actors.

It can be argued that there is no such a thing as a single "right" or universal definition of institutions and different conceptions can be used according to the purpose or objective of the particular analytical framework. However, it is of crucial importance that the reader understands what it means in the specific context she is analyzing. Therefore, it is of no importance that Varieties of Capitalism diverges from the NIE's concept of institutions; but it is an important drawback that Hall and Soskice do not provide a clear meaning of the term and use it inconsistently in their framework.

\section{CONCLUSION}

The link between national institutions and economic performance and the development of capitalism has preoccupied researchers from variety of disciplines for many years. Given the complexity and multidimensionality of the process of historical development and given the continuous institutional and economic change, it is extremely difficult to provide an analytical framework integrating institutional analysis into economic history. This paper attempted to review the concept of institutions and its relationship to capitalist development as used in the field of NIE and compared it to the use of the concept by Hall and Soskice. It concludes that by specifying exactly what institutions are, how and why they should be differentiated from organizations, and how they influence transaction and production costs, Douglass North and the NIE articulate the role of institutions in understanding economic development. Hall and Soskice attempt to follow the definition of North; however, their analysis reveals a broad and varied use of the concept which ignores one of the key elements of institutions - enforcement. Moreover, Hall and Soskice fail to provide a clear and consistent differentiation between institutions and organizations which leads to confusions in terms of understanding their specific role in and impacts on the process of development of the two types of economies that the authors define. There is a consensus among researchers regarding the great importance of institutions in capitalist development. However, a varied, imprecise and inconsistent use of the concept can also lead to an overestimation of the role of institutions and conversely underestimation of the role of organizations in capitalist development.

\section{REFERENCES}

1. Hall, P. and D. Soskice (2001). An Introduction to Varieties of Capitalism in P. Hall and D. Soskice, eds., Varieties of Capitalism. Oxford University Press: Oxford.

2. Harriss, J., J. Hunter and C. M. Lewis (1995). The New Institutional Economics and Third World Development. Routledge: New York.

3. Clague, C. P. Keefer, S. Knack and M. Olson (1997). Institutions and Economic Development: Growth and Governance in Less-Developed and Post-Socialist Countries. The Johns Hopkins University Press:

Baltimore, Maryland. 
4. Nabli, M.K. and J.B. Nugent (1989). The New Institutional Economics and Its Applicability to Development World Development, Vol. 17, No. 19, pp. 1333-1347.

5. North, D. C. (1990). Institutions, Institutional Change and Economic Performance. Cambridge University Press: New York.

6. $\quad$ Preston, P. W. (1996). Development Theory: An Introduction. Blackwell Publishers Ltd: Cambridge: Massachusetts.

7. Yeager, T. J. (1998). Institutions, Transition Economies, and Economic Development. Westview Press: Boulder, Colorado.

\section{NOTES}




\section{NOTES}

